\title{
Influência da migração na prevalência de marcadores sorológicos de hepatite B em comunidade rural. 1- Análise da prevalência segundo local de nascimento*
}

\author{
Influence of migration on prevalence of serological hepatitis $B$ markers in a rural \\ community. 1- Analysis of prevalence by birthplace
}

Afonso D. C. Passos**, Uilho A. Gomes"*, José F. de C. Figueiredo***, Margarida M. P. do Nascimento***, Jaqueline M. de Oliveira****, Ana M. C. Gaspar ${ }^{* * * *}$, Clara F. T. Yoshida****

\begin{abstract}
PASSOS, A. D. C. et al. Influência da migração na prevalência de marcađores sorológicos de hepatite B em comunidade rural. 1- Análise da prevalência segundo local de nascimento. Rev. Saúde Pública, 27(1): 30-5, 1993. Como parte de uma investigação epidemiológica de campo sobre hepatite B num município de características rurais do Estado de São Paulo, Brasil, foi estudada a distribuição de marcadores sorológicos dessa doença segundo à área de residência e o local de nascimento dos indivíduos. Para o município estudado como um todo, a prevalência encontrada para um ou mais dos marcadores sorológicos de hepatite B foi de $7,7 \%$, com os habitantes rurais apresentando risco mais elevado que os urbanos $(9,8 \%$ e $4,9 \%$, respectivamente). A análise da positividade, de acordo com o local de nascimento, mostrou valores mais altos entre os migrantes provenientes de outros Estados do País $(15,8 \%)$, seguidos dos oriundos de outros municípios de São Paulo $(9,2 \%)$ : entre os nascidos no município estudado e, particularmente em Ribeirão Preto, centro urbano de localização próxima ao mesmo, observaram-se as menores prevalências $(5,2 \%$ e $2,5 \%$, respectivamente). Discute-se a importância de se analisar em estudos epidemiológicos, a procedência dos indivíduos, variável capaz de influir na história natural da hepatite $\mathrm{B}$ numa comunidade, e, eventualmente, explicar diferenças nas distribuições de marcadores dessa infecção em populações aparentemente semelhantes.
\end{abstract}

Descritores: Hepatite B, epidemiologia. Migração interna. Distribuição espacial.

\section{Introduçăo}

Muito embora movimentos populacionais desempenhem papel de grande relevância na disseminação de diferentes doenças infecto-parasitárias, a possibilidade de que os mesmos apresentem algum significado na história natural da hepatite $B$ não tem merecido o devido destaque. Enquanto referências esporádicas a esse respeito têm surgido na li-

\footnotetext{
* Parte da Tese de Doutorado apresentada ao Deparamento de Medicina Social da Faculdade de Medicina de Ribeirão Preto da Universidade de São Paulo, 1991.

* Departamento de Medicina Social da Faculdade de Medicina de Ribeirão Preto da Universidade de São Paulo.

*** Departamento de Clínica Médica da Faculdade de Medicina de Ribeirão Preto - USP - São Paulo, SP - Brasil.

**** Centro de Referência Nacional para Hepatites Virais/ FIOCRUZ - Rio de Janeiro - Brasil.
}

Separatas/Reprints: A. D. C. PASSOS - Av. Bandeirantes, 3900 - 14049-900 - Ribeirāo Preto, SP - Brasil.

Edição subvencionada pela FAPESP. Processo Medicina 93/0208-5. teratura internacional 13,4,7,8,9,10,13,14,15,16, no Brasil destaca-se apenas a demonstração de distribuiçð̃es diferenciadas de subtipos de HBsAg nas diversas macrorregiōes geográficas, apontando para a importância das variadas colonizaçð̌es que se estabeleceram nestas áreas e dos movimentos migratórios que para elas têm se dirigido ${ }^{2}$. Como parte de uma ampla investigação epidemiológica sobre hepatite B levada a efeito em Cássia dos Coqueiros, município de 2.800 habitantes localizado a $80 \mathrm{Km}$ de Ribeirão Preto, foi possível estudar a influência exercida pela migração na distribuição de marcadores sorológicos da doença, o que constitui o motivo do presente trabalho.

\section{Material e Método}

Foi definida como população de estudo a totalidade dos moradores do Município de Cássia dos Coqueiros, Estado de São Paulo, com idade igual ou superior a um ano. Após a realização de um cadastramento que incluiu toda a área municipal, os habitantes foram convidados a participar da inves- 
tigação, tendo sido previamente informados dos seus objetivos e metodologia. A cada participante foi aplicado questionário específico, com questőes relativas ao domicílio, características gerais dos moradores e fatores de risco para hepatite B. Simultaneamente, foi coletada uma amostra de 10 $\mathrm{ml}$ de sangue para realização de testes imunoenzimáticos para detecção dos marcadores sorológicos HBsAg, anti-HBs e anti-HBc, utilizandose reativos e metodologia padronizada pelo Centro de Referência Nacional para Hepatites Virais (CRNHV), da Fundação Instituto Oswaldo Cruz. Mais detalhes da metodologia concemente à realização e interpretação dos exames sorológicos já foram publicados anteriormente ${ }^{11}$.

A análise estatística das tabelas bidimensionais foi realizada mediante a aplicação do teste quiquadrado. Nas tabelas multidimensionais a análise foi realizada pela aplicação do modelo log linear, utilizando-se o programa GLIM 3.77 - Royal Statistical Society ${ }^{12}$. Todas as tabelas foram submetidas inicialmente a um teste de independência mútua entre as variáveis. Caso este teste revelasse associação estatisticamente significante, procedia-se à realização dos testes de independência parcial. Em todas as situações o limite de significância para rejeição da hipótese de nulidade foi fixado em $5 \%$.

\section{Resultados}

As Figuras 1, 2 e 3 mostram a distribuição da população de Cássia dos Coqueiros e da amostra estudada segundo as variáveis área de residência, sexo e idade. No total foram estudadas 1.951 pessoas, representando $69,7 \%$ do total de habitantes, correspondendo a $78,3 \%$ dos moradores urbanos $e$ $64,7 \%$ dos rurais, observando-se predomínio da participação feminina nas duas áreas e em pratica-

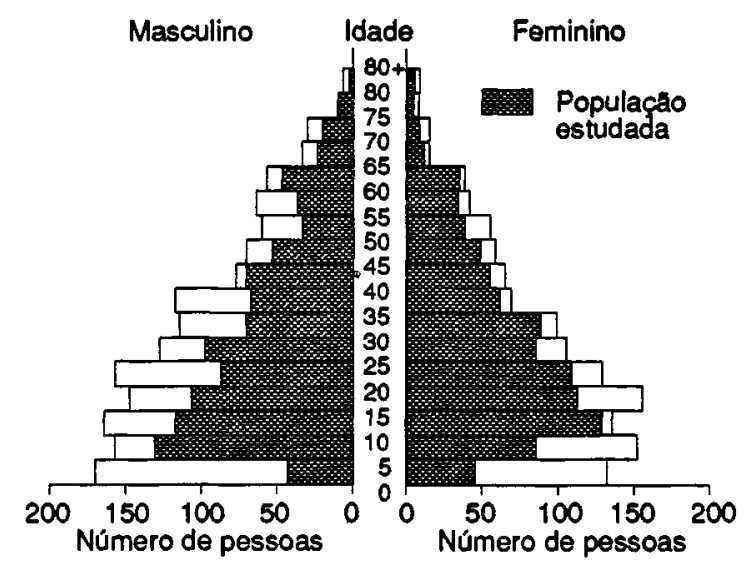

Figura 1. Distribuição da população total e da amostra estudada segundo sexo e idade. Cássia dos Coqueiros, SP. mente todas as faixas etárias. Observando-se as Figuras visualizam-se importantes diferenças na estrutura populacional das zonas urbana e rural. $\mathrm{Na}$ pirâmide que representa o total do Município (Fig. 1), o grande alargamento verificado na base e nos segmentos correspondentes aos adultos jovens reflete a distribuição populacional encontrada na área rural (Fig. 3), ocultando um padrão urbano de base mais estreitada e de distribuição consideravelmente mais homogênia (Fig. 2).

A representação da distribuição dos marcadores segundo a idade de ocorrência (Tabela 1) mostra prevalência total de $7,74 \%$, com valores progressivamente maiores à medida que avança a faixa etária - $\left[\mathrm{x}^{2}(8 \mathrm{gl})=33,20 ; \mathrm{p}=0,0001\right]$. As prevalências dos diferentes marcadores alcançaram valores iguais a $7,7 \%$ para o anti-HBc, 1,7 para o anti-HBs e $0,1 \%$ para o HBsAg. Não se observaram diferenças significativas no que diz respeito à distribuição de marcadores segundo a variável sexo.

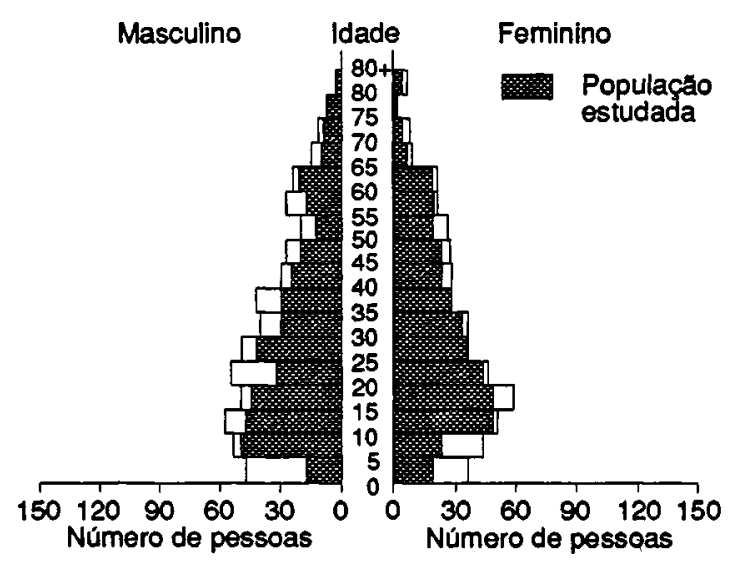

Figura 2. Distribuição da população urbana e da amostra estudada segundo sexo e idade. Cássia dos Coqueiros, SP.

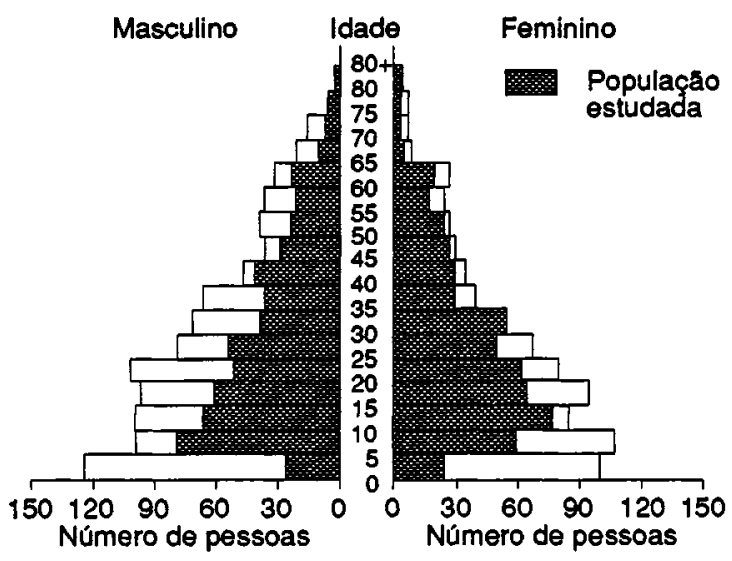

Flgura 3. Distribuição da população rural e da amostra estudada segundo sexo e idade. Cássia dos Coqueiros, SP. 
Em relação à área đe residência e faixa etária (Fig. 4), a distribuiç̧̃o dos marcadores mostra ausência de positividade apenas entre os habitantes urbanos abaixo de 5 anos de idade. Variaçōes sem um padrão definido ocorrem até os 15 anos, após o que a prevalência assume valores constantemente mais elevados entre os habitantes da zona rural. Incluindo-se na análise estatística a variável sexo, os testes de independência parcial mostram as seguintes associaçōes: a) entre positividade e idade, dentro de cada classificação de sexo e área - $\left[\mathrm{x}^{2}(16 \mathrm{gl})=33,87(\mathrm{p}<\right.$ $0,01)]$; b) entre positividade e área, dentro de cada classificação de sexo e idade $-\left[\mathrm{x}^{2}(14 \mathrm{gl})=\right.$ $24,12(\mathrm{p}<0,05)]$.

A distribuição de marcadores nos habitantes urbanos e rurais, segundo o tempo de residência em Cássia dos Coqueiros (Tabela 2), mostra que, no total, as maiores prevalências são observadas nos moradores há mais de 50 anos $(12,8 \%)$ e nos há menos de 5 anos $(14,7 \%)$, o que se reflete em distribuiçð̃es diferenciadas nos meios urbanos e rural. Enquanto no primeiro as variaçōes são menos expressivas e $\mathbf{a} \cdot$ maior prevalência se verifica nos moradores há mais de 50 anos $(9,0 \%)$, no segundo ocorrem variações mais acentuadas e com prevalências mais elevadas nos dois extremos. Os testes de independência parcial mostram as seguintes associações: a) entre positividade e tempo de residência, dentro de cada classificação de área - $\left[\mathrm{x}^{2}(8 \mathrm{gl})\right.$ $=42,75-(\mathrm{p}<0,001)]$; b) entre positividade e área, dentro de cada classificação de tempo de residência - $\left[\mathrm{x}^{2}(5 \mathrm{gl})=16,49(\mathrm{p}<0,01)\right]$.

A análise da distribuição das prevalências de marcadores segundo a área de residência e o local

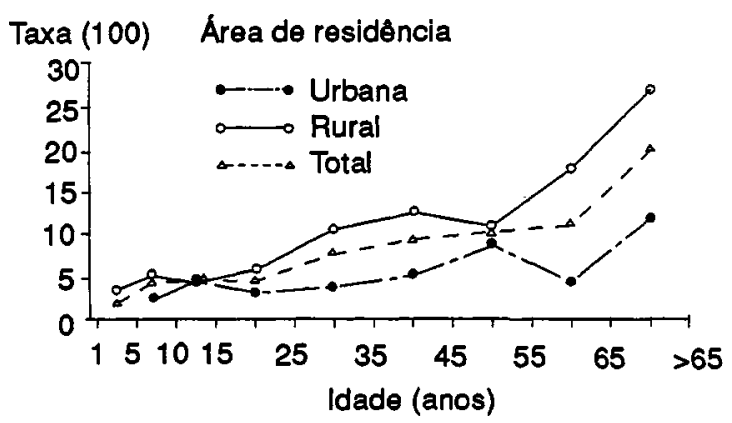

Figura 4. Prevalência de marcadores sorológicos de hepatite $B$ segundo a área de residência $e$ a idade da população estudada. Cássia dos Coqueiros, SP.

Tabela 1. Prevalência de diferentes marcadores sorológicos de hepatite B segundo a idade. Cássia dos Coqueiros, SP.

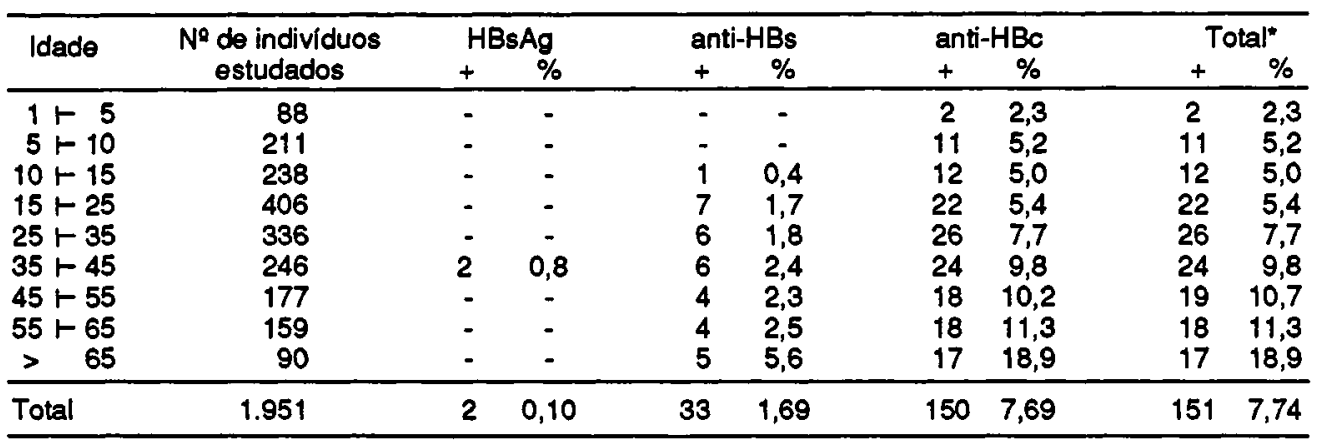

- Individuos com um ou mais marcadores presentes.

Tabela 2. Prevalência de marcadores sorológicos de hepatite $B$ segundo a área e o tempo de residência no município de Cássia dos Coqueiros, SP*.

\begin{tabular}{|c|c|c|c|c|c|c|c|c|c|c|}
\hline \multirow{3}{*}{ Tempo de residência } & \multirow[b]{3}{*}{ Positiv. } & \multicolumn{6}{|c|}{ Área } & & & \\
\hline & & \multicolumn{3}{|c|}{ Urbana } & \multicolumn{3}{|c|}{ Rural } & \multicolumn{3}{|c|}{ Total } \\
\hline & & $n$ & + & $\%$ & $\mathrm{n}$ & + & $\%$ & $n$ & + & $\%$ \\
\hline $\begin{array}{r}<5 \\
6-10 \\
11-20 \\
21-50 \\
51 e+\end{array}$ & & $\begin{array}{r}96 \\
135 \\
204 \\
292 \\
89\end{array}$ & $\begin{array}{r}7 \\
10 \\
7 \\
8 \\
8\end{array}$ & $\begin{array}{l}7,3 \\
7,4 \\
3,4 \\
2,7 \\
9,0\end{array}$ & $\begin{array}{r}264 \\
180 \\
277 \\
323 \\
90\end{array}$ & $\begin{array}{l}46^{\circ} \\
10 \\
17 \\
23 \\
15\end{array}$ & $\begin{array}{r}17,4 \\
5,6 \\
6,1 \\
7,1 \\
16,7\end{array}$ & $\begin{array}{l}360 \\
315 \\
481 \\
615 \\
179\end{array}$ & $\begin{array}{l}53 \\
20 \\
24 \\
31 \\
23\end{array}$ & $\begin{array}{r}14,7 \\
6,3 \\
5,0 \\
5,0 \\
12,8\end{array}$ \\
\hline Total & & 816 & 40 & 4,9 & 1.134 & 111 & 9,8 & 1.950 & 151 & 7,7 \\
\hline
\end{tabular}

- Excluido 1 indivíduo com tempo de residência desconhecido (área urbana). 
de nascimento (Tabela 3 ) revela que os mais altos valores totais são encontrados nos indivíduos provenientes de outros Estados do País $(15,8 \%)$, seguidos dos oriundos de outros municípios do Estado de São Paulo $(9,2 \%)$. Entre os naturais de Cássia dos Coqueiros e, particularmente, de Ribeirão Preto, as prevalências assumem valores consideravelmente mais baixos $(5,2 \%$ e $2,5 \%$, respectivamente). O mesmo padrão é verificado entre os residentes das áreas urbana e rural, muito embora, entre os habitantes rurais, as prevalências observadas em indivíduos provenientes de outros Estados e de outros municípios sejam acentuadamente mais elevadas do que entre os urbanos ( $18,3 \%$ contra $7,5 \%$ e $11,1 \%$ contra $5,7 \%$, respectivamente). Esta diferença para mais entre os residentes em área rural se reduz de modo acentuado entre os naturais de Cássia dos Coqueiros $(5,7 \%$ contra 4,7\%) e desaparece entre os nascidos em Ribeirão Preto $(2,4 \%$ e 2,7\%). Agrupando-se os nascidos em Cássia dos Coqueiros e Ribeirão Preto, os testes de independência parcial mostram as seguintes associaçðes: a) entre positividade e área, dentro de cada categoria de local de nascimento $\left.\left[x^{2}(3 \mathrm{gl})=9,67(\mathrm{p}<0,05)\right] ; \mathrm{b}\right)$ entre positividade e local de nascimento, dentro de cada categoria de área - $\left[\mathrm{x}^{2}(4 \mathrm{gl})=38,72(\mathrm{p}<0,001)\right]$.

Estudando-se as mesmas variáveis da Tabela 3, reunidas em apenas dois grupos (nascidos em Cássia dos Coqueiros ou Ribeirão Preto e em outros municípios ou Estados), observa-se (Tabela 4) uma prevalência 2,6 vezes superior entre os últimos $(12,0 \%$ e $4,6 \%)$. Esta grande diferença fazse de modo particular às custas dos residentes em área rural, onde a prevalência entre os nascidos em outros municípios ou Estados alcança um valor de 2,9 vezes maior que a observada entre originários de Cássia dos Coqueiros ou Ribeirão Preto (14,5\% e 5,0\%, respectivamente). Entre os moradores da área urbana também ocorre uma prevalência mais alta naqueles nascidos em outros municipios/ Estados, embora com uma diferença consideravelmente menor $(6,3 \%$ e $4,3 \%)$. Os testes de independência parcial entre as variáveis revelam as seguintes associaçðes: a) entre positividade e área, dentro de cada categoria de local de nascimento $\left.\left[\mathrm{x}^{2}(2 \mathrm{gl})=11,64(\mathrm{p}<0,01)\right] ; \mathrm{b}\right)$ entre positividade e local de nascimento, dentro de cada categoria de área - $\left[\mathrm{x}^{2}(2 \mathrm{gl})=30,14(\mathrm{p}<0,001)\right]$.

\section{Discussáo}

A pirâmide populacional correspondente à totalidade do município de Cássia dos Coqueiros mostra um padrão claramente mais alargado nos segmentos etários mais jovens, o que reflete elevada taxa de natalidade e constitui característica que se faz presente em populaçōes de menor nível socioeconômico. A comparação das pirâmides representativas das áreas urbana e rural evidencia padrões bastante diferenciados. Enquanto a segun-

Tabela 3. Prevalência de marcadores sorológicos de hepatite B segundo a área de residência e o local de nascimento. Cássia dos Coqueiros, SP.

\begin{tabular}{|c|c|c|c|c|c|c|c|c|c|c|}
\hline \multirow{3}{*}{ Local de nascimento } & \multirow[b]{3}{*}{ Positiv. } & \multicolumn{6}{|c|}{ Área } & & & \\
\hline & & \multicolumn{3}{|c|}{ Urbana } & \multicolumn{3}{|c|}{ Rural } & \multicolumn{3}{|c|}{ Total } \\
\hline & & $n$ & + & $\%$ & $n$ & + & $\%$ & $n$ & + & $\%$ \\
\hline $\begin{array}{l}\text { Cássia dos Coqueiros } \\
\text { Ribeirão Preto } \\
\text { Outro municipio Eșt. S. Paulo } \\
\text { Outro estado } \\
\text { Prejudicado }\end{array}$ & & $\begin{array}{r}449 \\
113 \\
175 \\
80 \\
-\end{array}$ & $\begin{array}{r}21 \\
3 \\
10 \\
6 \\
-\end{array}$ & $\begin{array}{r}4,7 \\
2,7 \\
5,7 \\
7,5 \\
-\end{array}$ & $\begin{array}{r}435 \\
123 \\
305 \\
268 \\
3\end{array}$ & $\begin{array}{r}25 \\
3 \\
34 \\
49 \\
-\end{array}$ & $\begin{array}{r}5,7 \\
2,4 \\
11,1 \\
18,3 \\
-\end{array}$ & $\begin{array}{r}884 \\
236 \\
480 \\
348 \\
3 \\
\end{array}$ & $\begin{array}{r}46 \\
6 \\
44 \\
55 \\
-\end{array}$ & $\begin{array}{r}5,2 \\
2,5 \\
9,2 \\
15,8 \\
-\end{array}$ \\
\hline Total & & 817 & 40 & 4,9 & 1.134 & 111 & 9,8 & 1.951 & 151 & 7,7 \\
\hline
\end{tabular}

Tabela 4. Prevalência de marcadores sorologicos de hepatite B segundo a área de residência e o local de nascimento. Cássia dos Coqueiros, SP.

\begin{tabular}{|c|c|c|c|c|c|c|c|c|c|}
\hline \multirow{3}{*}{ Local de nascimento } & \multicolumn{6}{|c|}{ Área } & & & \\
\hline & \multicolumn{3}{|c|}{ Urbana } & \multicolumn{3}{|c|}{ Rural } & \multicolumn{3}{|c|}{ Total } \\
\hline & $n$ & + & $\%$ & $n$ & + & $\%$ & $n$ & + & $\%$ \\
\hline $\begin{array}{l}\text { Cássia dos Coqueiros e Ri- } \\
\text { beirão Preto } \\
\text { Outros municípios/estados } \\
\text { Prejudicado }\end{array}$ & $\begin{array}{c}562 \\
255 \\
-\end{array}$ & $\begin{array}{l}24 \\
16 \\
-\end{array}$ & $\begin{array}{l}4,3 \\
6,3 \\
-\end{array}$ & $\begin{array}{r}558 \\
573 \\
3\end{array}$ & $\begin{array}{l}28 \\
83 \\
-\end{array}$ & $\begin{array}{r}5,0 \\
14,5 \\
-\end{array}$ & $\begin{array}{r}1.120 \\
828 \\
3\end{array}$ & $\begin{array}{l}52 \\
99 \\
-\end{array}$ & $\begin{array}{r}4,6 \\
12,0 \\
- \\
\end{array}$ \\
\hline Total & 817 & 40 & 4,9 & 1.134 & 111 & 9,8 & 1.951 & 151 & 7,7 \\
\hline
\end{tabular}


da assemelha-se à observada para o total do $\mathrm{Mu}$ nicípio, a pirâmide da população urbana revela uma formatação em "barril", caracterizada por um acentuado estreitamento na sua base $e$ uma tendência lentamente decrescente à medida que se alcançam segmentos de maior idade. Esses achados refletem diferenças na dinâmica populacional nas duas áreas, expressas principalmente por menor concentração de crianças no meio urbano. A razão deste menor número de individuos jovens habitando a zona urbana deve ser, provavelmente, creditada a dois eventos demográficos distintos atuando na mesma direção. $O$ primeiro deles representado por uma taxa de natalidade mais reduzida entre os moradores da cidade, reproduzindo fenômeno universalmente observado de queda dos níveis de crescimento populacional associado à urbanização. Em segundo lugar, há que ser lembrado o fato de que o meio urbano local apresenta-se muito menos receptivo a afluxos migratórios, os quais se fazem na sua grande maioria para as áreas rurais e se caracterizam pelo aporte de măo de obra não especializada, que se dirige basicamente a atividades agrícolas. Fazendo da migraçăo uma estratégia de sobrevivência, esses contingentes populacionais pertencem invariavelmente a segmentos de baixo nível socioeconômico, entre os quais elevadas taxas de natalidade constituem uma característica frequientemente observada.

Conforme discutido em publicação anterior ${ }^{11}$, dois fatos chamam inicialmente a atenção no que diz respeito à distribuição dos marcadores sorológicos de hepatite $B$ na comunidade estudada. Em primeiro lugar, a importância da' inclusão do teste anti- $\mathrm{HBc}$ em estudos epidemiológicos, resultando imagem mais precisa da circulação deste agente viral numa comunidade. Um segundo achado merecedor de atenção diz respeito aos valores consideravelmente baixos de prevalência, situando Cássia dos Coqueiros em níveis comparáveis aos verificados fora das populações de risco da América do Norte, Europa Ocidental, Austrália e parte temperada da América do $\mathrm{Sul}^{14}$. Considerando-se as diferenças entre o nível de desenvolvimento socioeconômico dessas populações e da comunidade estudada, discutiu-se a hipótese de que a baixa prevalência em Cássia dos Coqueiros pudesse se dever ao seu relativo isolamento geográfico e à predominância de padrões de comportamento mais tradicionais e conservadores, características comuns às pequenas comunidades rurais do interior brasileiro 11 .

Por outro lado, a análise de prevalência dos marcadores de HVB revela niveis consistentemente mais elevadas entre os habitantes rurais, o que faz supor existir entre eles fatores de risco que se fazem presentes com maior intensidade do que no meio urbano e, aparentemente, contradiz a idéia de que a baixa prevalência global do Município possa se dever ao seu isolamento relativo e ao estilo de vida mais tradicional de seus moradores.

Conquanto as distribuições das zonas urbana $e$ rural mantenham padrōes assemelhados, com ascensão nas prevalências após tempos mais longos de moradia nas respectivas áreas, ressalta-se a discrepância entre elas quando se particularizam os indivíduos com tempo de residência inferior a 5 anos, situaçăo em que ocorre amplo predomínio da positividade entre os rurais. A impossibilidade de que isto possa ser explicado por uma maior concentração de marcadores em crianças rurais, com idade abaixo de 5 anos, toma lícito levantar a hipótese de que a presença da doença no $\mathrm{Mu}$ nicípio possa estar associada a movimentos migratórios, os quais, por se dirigirem mais amiúde para áreas rurais, fariam com que a prevalência detectada nessas regiós apresentasse valores mais elevados. Esta hipótese ganha consistência quando se analisa o local de nascimento dos positivos. Com efeito, as diferenças nas prevalências de marcadores entre os habitantes da cidade e do meio rural revelam-se mínimas entre os nascidos em Cássia dos Coqueiros e se tornan inexistentes entre os naturais de Ribeirăo Preto. Como estes últimos representam, com frequência, membros de núcleos familiares estabelecidos em Cássia dos Coqueiros, cujos nascimentos em Ribeirão Preto ocorrem em função de encaminhamento de situaçðes especiais ao Hospital das Clínicas ou de opção da familia por um serviço hospitalar, supõese que os nascidos nestas duas cidades apresentem semelhanças que permitam considerá-los como um agrupamento único e razoavelmente homogêneo. Ausentes neste agrupamento, as diferenças de prevalências entre as áreas urbana e rural manifestam-se com clareza entre os nascidos em outros municípios de São Paulo e, particularmente, entre os naturais de outros Estados.

Esses achados evidenciam o importante papel assumido por fenômenos migratórios na história natural da hepatite $B$, na comunidade estudada. Associação similar já foi discutida em algumas situaçð̃es na literatura internacional, merecendo atenção por diferentes razōes. Em primeiro lugar, pela possibilidade de representar uma fonte de transmissão da doença no local de estabelecimento dos migrantes, a partir de portadores cronicamente infectados. No município de Cássia dos Coqueiros tal possibilidade surge como remota, uma vez que a dispersão dos migrantes em atividades agrícolas na área rural acarreta pouca ou nenhuma chance de contacto próximo com os demais moradores do Município. Esta eventualidade, entretanto, assume importância em situaçð̃es em que grandes contingentes de migrantes, provenientes de áreas de alta 
prevalência, se estabelecem em cidades, tal como representado pelas centenas de milhares de asiáticos na California ${ }^{3,4,5}$. Ou então pela adoção, por famílias americanas, de crianças originárias da Coréia, India, Vietnã e América Latina ${ }^{3,7}$.

Uma segunda razão pela qual migrantes podem afetar a história natural da hepatite B diz respeito à possibilidade de aumentarem a prevalência da doença na população para a qual se dirigem, causando dificuldades na interpretação dos achados caso a associação da doença com a migração não seja considerada. Como exemplo, muito embora o vírus da hepatite $\mathrm{B}$ constitua inegável risco profissional entre pessoal médico e paramédico, o nascimento e/ou uma longa permanência em países estrangeiros já foram identificados como sendo tão ou mais importantes na positividade para a doença do que a própria atividade exercida por funcionários dentro do ambiente hospitalar ${ }^{6,9}$. De modo análogo, a análise do país de nascimento mostrou ser esta a variável associada ao aumento da incidência de carcinoma hepatocelular no Reino Unido, em anos recentes ${ }^{16}$. Percebe-se, com isso, a relevância de se considerar a procedência dos indivíduos quando da realização de estudos epidemiológicos sobre hepatite B. De importância evidente no estudo de Cássia dos Coqueiros, tal variável pode ser responsável por diferenças nas prevalências de marcadores sorológicos da moléstia, em localidades semelhantes, fenômeno não raramente observado e para o qual nem sempre existem explicações satisfatórias.

PASSOS, A. D. C. et al. [Influence of migration on prevalence of serological hepatitis B markers in a rural community. 1- Analysis of prevalence by birthplace]. Rev. Saúde Pública, 27: 30-5, 1993. Distribution of hepatitis B serological markers according to people's birthplaces and area of residence (urban/rural) was studied as a part of a field epidemiological research project carried out in Cássia dos Coqueiros, a small rural community of S. Paulo State, Brazil. The total prevalence of HBV markers was $7.7 \%$, with rural showing a higher risk than urban inhabitants $(9.8 \%$ as against $4.9 \%$, respectively). Analysis of prevalence according to people's birthplaces revealed the highest value among those from other Brazilian states (15.8\%), followed by people from other municipalities of the State of S. Paulo (9.2\%). Those bom in Cássia dos Coqueiros and particularly in Ribeirão Preto (the main city of the area, located 80 kilometers aw ay), showed the lowest values of prevalence $(5.2 \%$ and $2.5 \%$, respectively). The importance of studying people's birthplaces when field epidemiological studies on hepatitis B are carried out is stressed. This variable is considered to be capable of exercising an influence on the natural history of the disease in a community, and may even explain differences in the distribution of markers in apparently similar populations.
Keywords: Hepatitis B, epidemiology. Internal migration. Residence characteristics.

\section{Referências Bibliográficas}

1. AREVALO, J. A. \& AREVALO, M. Prevalence of hepatitis $B$ in an indigent, multiethnic community clinic prenatal population. J. Fam. Pract., 29: 615-9, 1989.

2. GASPAR, A. M. C. \& YOSHIDA, C. F. T. Geographic distribution of HBsAG subtypes in Brazil. Mem. Inst. Oswaldo Cruz, 82: 253-8, 1987.

3. GREENBLATT, M. \& KHOO, E. C. Incidence of hepatitis B carriers among adopted korean children. N. Engl. J. Med., 312: 1639, 1985.

4. GOLDERING, J. M. \& CASTLE, G. F. Prevalence of disease in southeast asian teenagers. Results of screening medical examination at a residential vocational training facility. J. Adolesc. Health Care, 4: 266-9, 1983.

5. GUNBY, P. Califomia battles perinatal hepatitis B. JAMA, 247: 1238, 1982.

6. HOVIG, B.; ROLLAG, H.; DAHL, O. Antibody to hepatitis $B$ surface antigen among employees in the National Hospital, Oslo, Norway: a prevalence study. Am. J. Epidemiol., 122: 127-34, 1985 .

7. JENISTA, J. A. \& CHAPMAN, D. Medical problems of foreign-born adopted children. Am. J. Dis. Child, 141: 298-302, 1987.

8. KALISH, S. B.; FISHER, B.; WALLEMARK, C. B.; CHMIEL, J. S.; PHAIR, J. P. Prevalence of antibody to hepatitis B virus in foreign-bom hospital employees. Am.J.Med., 83: 824-8, 1987.

9. KIEL, F. W. Brief report: influence of country of origin on prevalence of hepatitis B markers among employees in a small suburban hospital. Infect. Control, 7(11):554-6 1986.

10. LNDH, G.; WEILAND, O.; HANSSON, B. G.; SVEDMYR, A. Delta infection among patients with chronic hepatitis $\mathrm{B}$ in the Stockholm region. Infection, 12: 27 30,1984

11. PASSOS, A. D. C.; GOMES, U. A.; FIGUEIREDO, J. F. de C.; NASCIMENTO, M. P. do; OLIVEIRA, J. M. de; GASPAR, A. M. C.; YOSHIDA, C. F. T. Prevalência de marcadores sorológicos de hepatite $B$ numa pequena comunidade rural do estado de São Paulo, Brasil. Rev. Saúde Pública, 26: 119-24, 1992.

12. ROYAL STATISTICAL SOCIETY. The generalized linear interactive modelling system. Release 3.77 manual. Oxford, Numerical Algorithms Group, 1985.

13. STRUVE, J.; GIESECKE, J.; OLCEN, P.; SYDOW, M.; WEILAND, $O$. Prevalence of hepatitis B virus markers in Sweden: a community-based sero survay of 4,000 young Swedish adults. Am. J. Epidemiol., 135: 409-17, 1992.

14. U. S. DEPARTMENT OF HEALTH AND HUMAN SERVICES. Public Health Service. Centers for Disease Control. Hepatitis surveillance. Atlanta, Ca. 1989.29 p. (Report, 52).

15. WILSON, J. L.; BRUNSON, J.; JOHNSON, M. Health problems in indo-chinese immigrants. J. Fam. Pract., 12: 551-7, 1981 .

16. ZAMAN, S. N.; JOHNSON, P. J.; WILLIAMS, R. Hepatocelular carcinoma in immigrants to the United Kingdom. Q.J.Med., 60: 813-7, 1986.

Recebido para publicaçẫo em 3.7.1992 Reapresentado em 11.11.1992 Aprovado para publicação em 19.11.1992 